\title{
Remote Sensing of Salinity: The Dielectric Constant of Sea Water
}

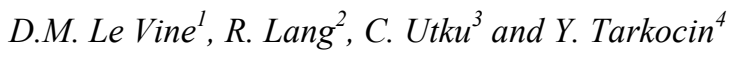 \\ ${ }^{1}$ Goddard Space Flight Center, Greenbelt, MD 20771; Email: David.M.LeVine@nasa.gov \\ ${ }^{2}$ The George Washington University, Washington, D.C. 20052: Email: lang@gwu.edu \\ ${ }^{3}$ Goddard Earth Science Technology Center, Goddard Space Flight Center, Greenbelt, MD 20771: Email: \\ Cuneyt.Utku@nasa.gov \\ ${ }^{4}$ TriTeck Solutions, 7617 Little River Turnpike, Annandale, VA 22003: Email: ytarkocin@tritecksol.com
}

\section{Abstract}

Global monitoring of sea surface salinity from space requires an accurate model for the dielectric constant of sea water as a function of salinity and temperature to characterize the emissivity of the surface. Measurements are being made at $1.413 \mathrm{GHz}$, the center frequency of the Aquarius radiometers, using a resonant cavity and the perturbation method. The cavity is operated in a transmission mode and immersed in a liquid bath to control temperature. Multiple measurements are made at each temperature and salinity. Error budgets indicate a relative accuracy for both real and imaginary parts of the dielectric constant of about $1 \%$.

\section{Introduction}

Ocean salinity is important for understanding ocean circulation and its relationship to climate and the global water cycle. Salinity, together with temperature, determines buoyancy, and the buoyancy driven thermohaline circulation moves large masses of water and heat around the globe. The Gulf Stream which moves warm water north and moderates the climate in Northern Europe is an example. Salinity is also an indicator of the global water cycle because surface salinity changes in response to the balance between the input and output freshwater flux (i.e. evaporation and precipitation).

Global monitoring of sea surface salinity from space is soon to become a reality. Changes in salinity modulate the emissivity of the ocean surface and the associated changes in thermal emission can be detected in the long wavelength end of the microwave spectrum (1.4 GHz, L-band). The primary instruments on SMOS which was launched in November, 2009 and Aquarius which is scheduled for launch in June, 2011, are L-band radiometer "pathfinders" whose goals include demonstrating global monitoring of sea surface salinity (SSS).

The measurement of SSS is quite challenging. The goal of Aquarius is to provide monthly maps with an accuracy of $0.2 \mathrm{psu}$ which is roughly equivalent to a radiometric accuracy of $0.1 \mathrm{~K}$. Achieving this accuracy requires a good model for the surface emissivity which in turn requires accurate knowledge of the dielectric constant of sea water.

In the mid-2000's Roger Lang and the first author proposed making precise measurements of the relative dielectric constant as a function of salinity and temperature at the center frequency $1.413 \mathrm{GHz}$ of the Aquarius and SMOS observations. The goal was an accurate model at this frequency with error bars, something missing in the past. Roger became the leader in this work and he and his graduate students at GWU solved problems and developed a sophisticated measurement facility [1].

\section{Measurement Approach}

After consideration of several possibilities, it was decided to use a microwave cavity operated in a perturbation mode, where the sea water is added in sufficiently small quantities to make a small perturbation on resonance. In this case, the dielectric constant can be determined from the change in the resonance frequency, $f$, and the change in the $\mathrm{Q}$ of the cavity as: 


$$
\epsilon^{\prime}-1=2 C \frac{\Delta f}{f}, \quad \epsilon^{\prime \prime}=C \Delta\left(\frac{1}{Q}\right)
$$

The method is similar to that employed by Ho and Hall [2] with the exception that the cavity is operated in the transmission mode (two coupling loops) rather than the reflection mode adopted by Ho and Hall. This was done to require less power and avoid the potential for heating of the sample that was reported with the earlier data. Figure 1 shows a schematic of the experimental setup and Figure 2 shows a photograph of the actual hardware. The cavity (Figure 1) is immersed in a liquid bath (water and ethylene glycol mixture) to control temperature. The cavity operates in the $\mathrm{TM}_{010}$ mode and the sample is introduced along the axis of the cylinder. The inner diameter of the tube is about $0.1 \mathrm{~mm}$. The network analyzer operates in transmission mode measuring $\mathrm{S}_{21}$.

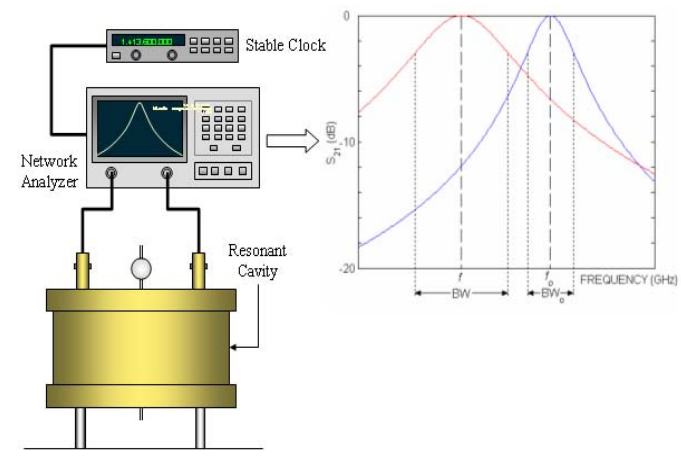

Figure 1: Schematic of experimental approach showing the resonant cavity, network analyzer and example resonant curves from which the $\mathrm{Q}$ and $\Delta f$ in Equation 1 are determined.

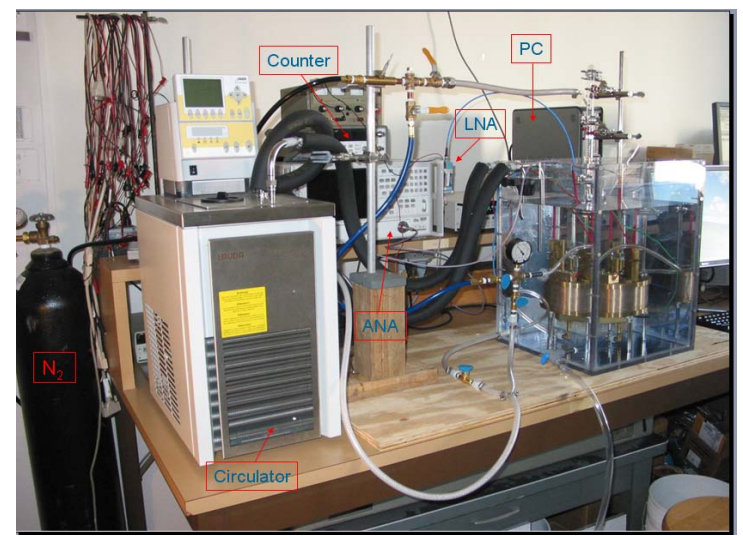

Figure 2: Photograph of the experiment as it existed in the laboratory at GWU. The cavity and liquid bath (water ethylene glycol mixture) are on the far right in a transparent Lexan container. This was later insulated to further improve temperature control. The device on the far left is the circulator that heats/cools and maintains the liquid temperature.

Given the desired accuracy, many problems had to be addressed. These include maintaining the flow of the water sample in the very small volume dictated by the requirements of the perturbation theory; maintaining stability of the measurement equipment against changes in the ambient temperature; and determining the calibration constant ( $C$ in Equation 1). 
A typical seawater experiment consists of several cycles. First, the cavity is brought to the desired temperature and allowed to stabilize. Next, 5 to 10 measurements are made with the tube empty. Then seawater is introduced into the tube. The sea water does not flow freely because of the small diameter of the tube and is forced with Nitrogen at about 2 atmospheres. The measurements are made at discrete values of temperature. After the measurement, the tube is cleaned with distilled water. The last step is to repeat the empty cavity measurements to confirm that the system has returned to its original state. The whole procedure can take from 1 to 3 hours.

\section{Results}

The goal of the measurement program is to obtain accurate measurements but also with meaningful error bars. The error bars account for noise (random variation between measurements) and systematic errors such as those associated with determining the calibration coefficient. Figure 3 below is an example of recent results. The figure shows the real part (left) and imaginary part (right) of the relative dielectric constant as a function of temperature for a salinity of 38.274 psu. For comparison, the red curves are the results from the model of Klein and Swift [3] which is based primarily on the earlier measurements of Ho [2,4]. Current results show about $1 \%$ difference in the real part and about $2 \%$ difference in the imaginary part of the dielectric constant compared to the Klein and Swift results. Work is continuing to obtain measurements over a complete range of temperatures and salinity representative of the seawater in the open ocean and to improve accuracy which currently is about $1.3 \%$ or less.
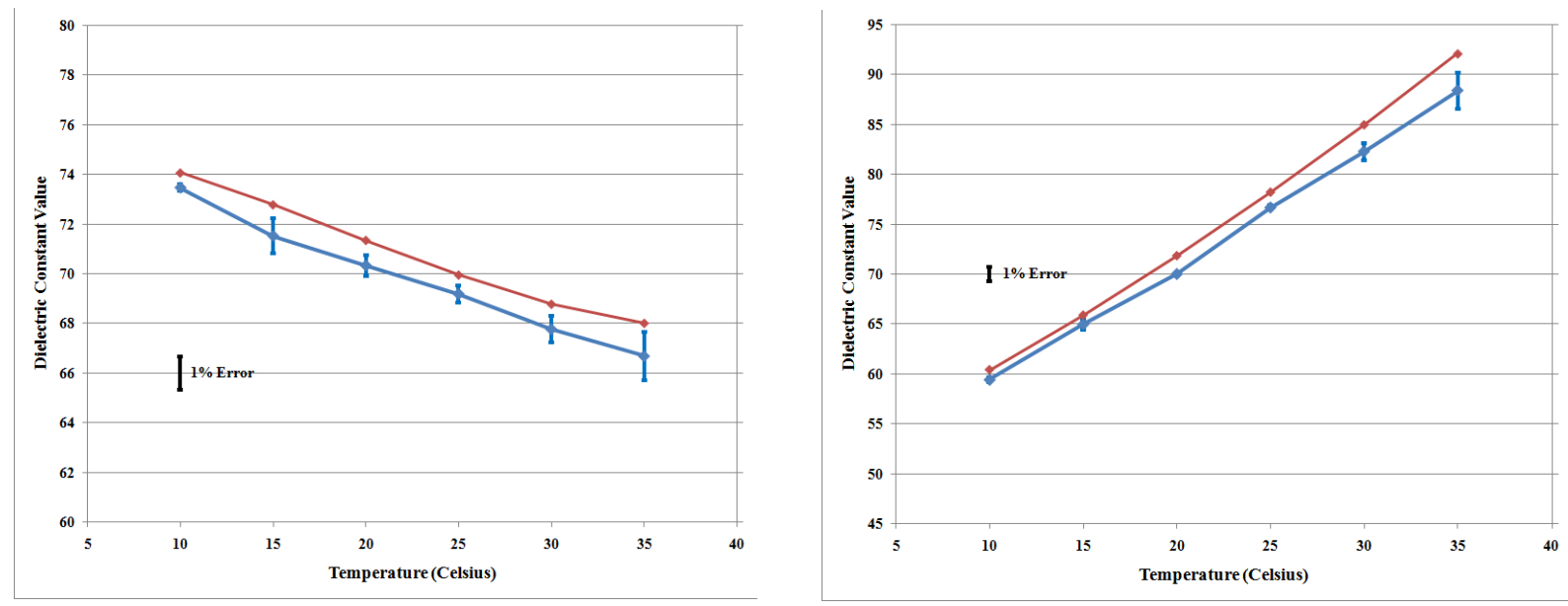

Figure 3: The real part (left) and imaginary part (right) of the dielectric constant vs temperature

\section{References}

1. R. Lang, Cuneyt Utku, Yalcin Turkocin and David Le Vine, "Accurate Measurements of the Dielectric Constant of Seawater at L Band,” NASA Technical Memorandum, NASA TM-2010-215861, 2010.

2. W.A. Ho and W.F. Hall, "Measurements of the dielectric properties of seawater of seawater and $\mathrm{NaCl}$ solutions at $2.65 \mathrm{GHz}$, “J. Geophys. Res., Vol. 78, pp. 6301-6315, 1973.

3. L. Klein and C. Swift, "An improved model for the dielectric constant of seawater at microwave frequencies, " IEEE Trans. Antennas and Propagation, Vol. 25, pp. 104-111, 1977.

4. W.A. Ho, W. Love and M.J. van Melle, "Measurements of the dielectric properties of sea water at $1.43 \mathrm{GHz}$," NASA Contract Report., CR-2458, 1974. 